\title{
Respiratory failure from an upper gastrointestinal series
}

A 67-year-old male was brought to hospital (Linda University Medical Center, Loma Linda, CA, USA) after being hit by a car. The patient underwent surgery for a fractured acetabulum. The post-operative course was complicated by 7 days of ileus. An upper gastrointestinal (GI) series (i.e. barium swallow) with small bowel follow-through was requested. There were no immediate complications during the upper GI series. The supine abdominal radiograph at $8 \mathrm{~h}$ post-upper GI series (fig. 1) showed relatively normal lungs and contrast within the stomach and proximal small bowel. $15 \mathrm{~h}$ after the upper GI series, the patient complained of shortness of breath with a respiratory rate of 14 breaths $\cdot \mathrm{min}^{-1}$ and an arterial oxygen saturation measured by pulse oximetry of $99 \%$. The patient was reassured. The supine abdominal radiograph at $16 \mathrm{~h}$ post-upper GI series (fig. 1) showed consolidation within the right lung. A contrast chest computed tomography scan was then performed (fig. 2) and showed hyperdense round irregular foci within the lung with surrounding consolidation. The densities within the lung represented aspirated barium contrast after the upper GI series while the patient was in his room. Due to the severe ileus, the contrast agent did not pass through the stomach
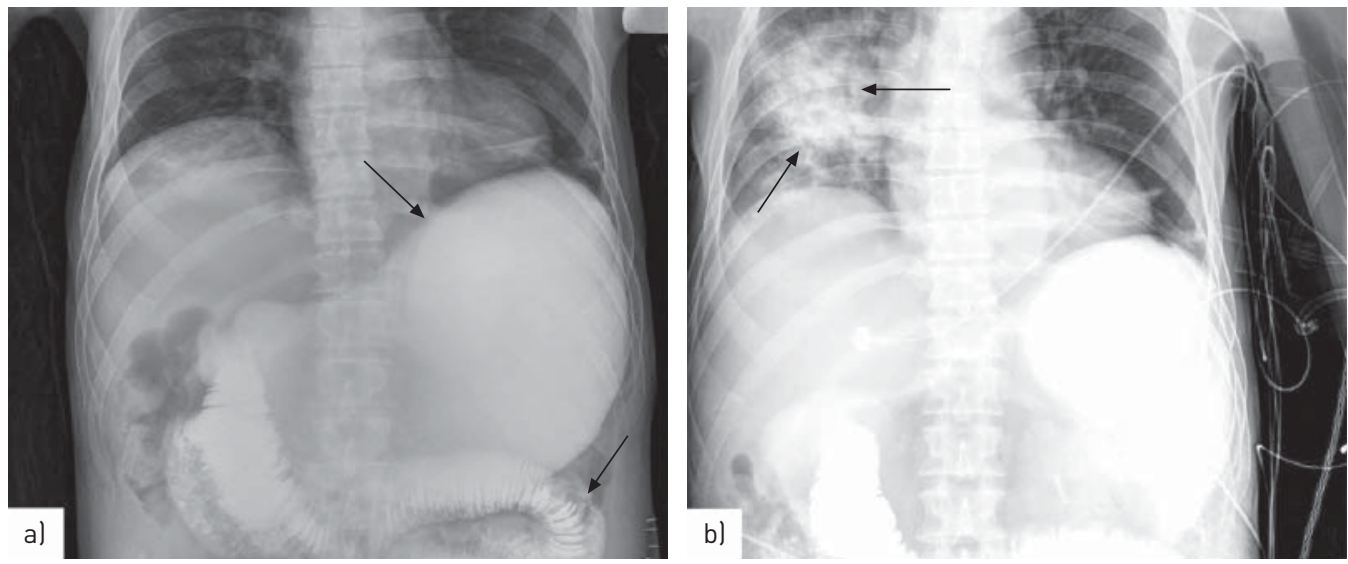

FIGURE 1 Supine abdominal radiograph from a 67-year-old male a) $8 \mathrm{~h}$ and b) $16 \mathrm{~h}$ following upper gastrointestinal series (barium swallow). a) The lungs are clear of focal consolidation and there is a large amount of contrast within the stomach and proximal small bowel (arrows). b) There is new dense consolidation of the visualised right lung (arrows).

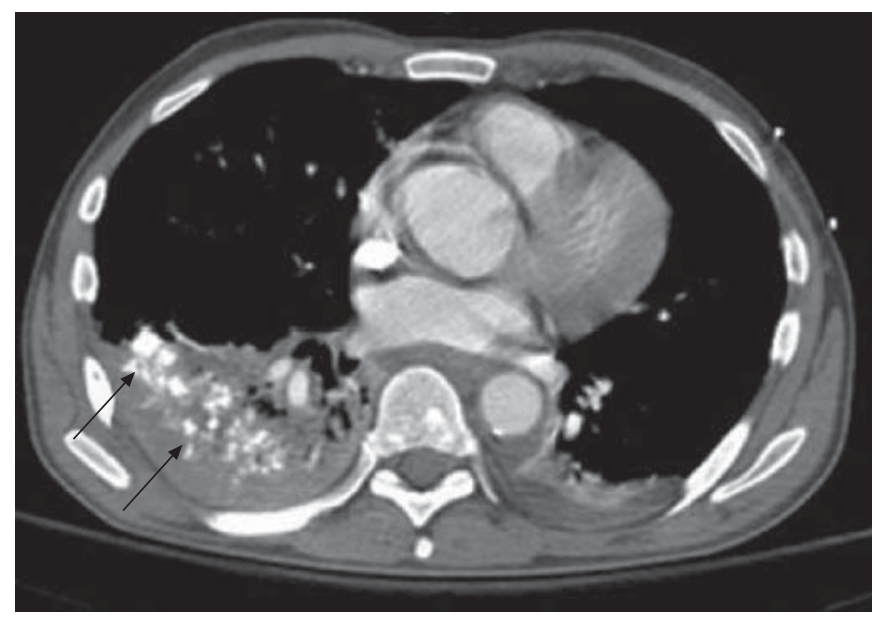

FIGURE 2 A contrast chest computed tomography scan from a 67-year-old male with respiratory failure 1 day after upper gastrointestinal series (barium swallow). There are hyperdensities within the right lower lobe bronchi (arrows) representing aspirated barium with surrounding consolidation. 
and remained at a location susceptible to aspiration. The patient went on to suffer acute respiratory failure as a result of acute pneumonitis and eventually had a tracheostomy tube inserted. This demonstrates the importance of aspiration precautions for patients with ileus.

@ERSpublications

Risk of aspiration needs to be accounted for in patients with ileus who have had oral contrast ingestion http://ow.ly/vyKw3

Naseem Helo, Daniel Hoss and Mark Girguis

Dept of Radiology, Loma Linda University Medical Center, Loma Linda, CA, USA.

Correspondence: Naseem Helo, Dept of Radiology, Loma Linda University Medical Center, 11234 Anderson St, Loma Linda, 92354 CA, USA. E-mail: Nhelo@llu.edu

Received: March 132014 | Accepted: March 172014

Conflict of interest: None declared.

Provenance: Submitted article, peer-reviewed.

\section{An unexpected discovery complicating sicca syndrome}

During a routine check-up, retro-pneumoperitoneum was discovered on the lowest slices of a computed tomography (CT) chest scan in a 77-year-old female patient who was known to have Sjögren's syndrome with lymphocytic interstitial pneumonia (fig. 1a). She had initially been treated with prednisolone that improved sicca signs and cough. The patient, who had no prior history of digestive problems, complained of abdominal discomfort and distension. She was afebrile, haemodynamically stable and bowel movement was unchanged. Abdominal examination eliminated signs of peritonism.

The abdominal CT scan, which showed thickening of the wall of the descending colon, contained multiple air-filled cysts (black arrows) consistent with pneumatosis cystoides intestinalis (PCI) (fig. 1b). There was free air in the wall of the caecum, colon and adjacent mesos (fig. 1c).

PCI is a rare condition characterised by multilocular pneumatocysts within the wall of the lower digestive tract. To date, association with a systemic connective tissue disease (CTD) such as Sjögren's syndrome has only been reported twice [1]. Previous studies have mostly been of scleroderma-related conditions [2]. Corticosteroids are often used in CTD and appear to increase the risk of PCI [3]. Conservative treatment is often sufficient in stable patients with limited symptoms. Antibiotic agents (e.g. metronidazole) and the tapering of immunosuppressive drugs seem to improve clinical and radiological findings. Intestinal rest, parenteral nutrition, electrolyte supplementation and inhaled oxygen are also recommended [3].
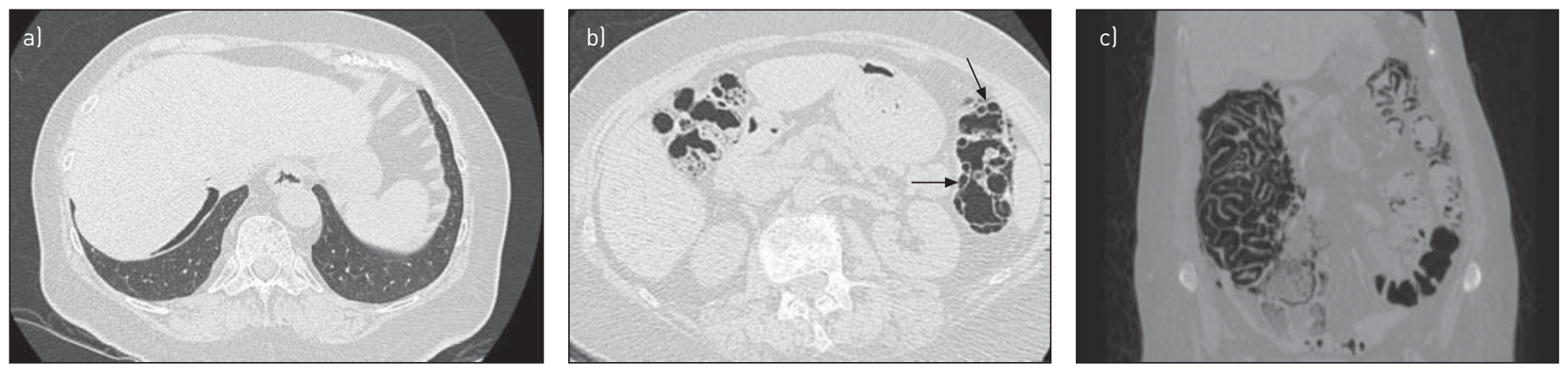

FIGURE 1 a) Computed tomography (CT) chest scan showing retro-pneumoperitoneum. b) Abdominal CT scan showing thickening of the wall of the descending colon, which contained multiple air-filled cysts (black arrows). c) Coronal CT reconstruction showing free air in the wall of the caecum, colon and adjacent mesos. 